\title{
MaRING2: A Positive Regulator of an Abscisic Acid-dependent Response to Cold Stress from Banana Fruit
}

\author{
Jiao Chen ${ }^{1}$, De-bao Yuan', Chao-zheng Wang, Yi-xing Li, and Fen-fang Li \\ Hainan Key Laboratory of Banana Genetic Improvement, Haikou Experimental station, Chinese \\ Academy of Tropical Agricultural Sciences, Haikou 570102, China
}

\author{
Ke-qian Hong \\ Key Laboratory for Postharvest Physiology and Technology of Tropical Horticultural Products of \\ Hainan Province, South Subtropical Crop Research Institute, Chinese Academy of Tropical, \\ Agricultural Sciences, Zhanjiang 524088, China \\ Wang-jin Lu \\ State Key Laboratory for Conservation and Utilization of Subtropical Agro-Bioresources/Guangdong \\ Key Laboratory for Postharvest Science, College of Horticultural Science, South China Agricultural \\ University, Guangzhou 510642, China
}

\begin{abstract}
AdDITIONAL INDEX WORDS. RING finger protein, abiotic stress, overexpression, transgenic tobacco
Abstract. Many reports indicate that an abundance of really interesting new gene (RING) play key roles in regulating defense responses against abiotic and biotic stresses in plants. In this study, the cloning and functional characterization of a RING gene, MaRING2, in banana (Musa acuminata) fruit are reported. MaRING2 belongs to the NEP1-interacting protein (NIP) RING-H2 finger protein family. Gene expression profiles revealed that $M a R I N G 2$ was cold responsive and induced by abscisic acid (ABA) treatment during cold storage. In this study, the MaRING2 under control of the Cauliflower mosaic virus 35S (CaMV 35S) promoter was transformed to tobacco (Nicotiana benthamiana) using agrobacterium (Agrobacterium tumefaciens)-mediated transformation. The resultant MaRING2overexpressing transgenic plants (35S:MaRING2) exhibited significantly increased tolerance to low temperatures and were hypersensitive to exogenous ABA in terms of germination and early seedling growth. In addition, overexpression of MaRING 2 enhanced the expression of stress-responsive genes under normal (before cold stress) or cold conditions. These results demonstrate the biological role of MaRING2 in conferring cold tolerance. Taken together, these results suggest that MaRING2, a C3H2C3-type RING protein, is a positive regulator of the ABA-dependent stress response.
\end{abstract}

Plants are frequently subjected to abiotic environmental stresses, such as low temperatures, drought, and high salinity. These conditions have significant detrimental effects on growth and crop yields. To address various environmental stresses, plants execute some physiological and metabolic responses that are normally mediated by the up-regulation of stress-regulated gene expression (Thomashow, 1999; Xiong et al., 2002; Xiong and Zhu, 2002; Zhu, 2001). These induced genes either directly protect plants against stresses by the production of important metabolic proteins (functional proteins) or regulate the genes for signal transduction in the stress response (regulatory proteins). It is of immense importance to elucidate the exact function of genes involved in signal transduction and their regulation to understand their responses to different environmental stresses in plants (Cui et al., 2002; Ronald and Leung, 2002).

Zinc finger protein genes constitute a large and diverse gene family. A number of zinc finger proteins have been found to be involved in abiotic and biotic stress responses (Cui et al., 2002; Huang et al., 2005; Kim et al., 2001; Mukhopadhyay et al.,

Received for publication 24 Mar. 2015. Accepted for publication 8 Apr. 2015. This work was supported by the National Natural Science Foundation of China (Project No. 31301823) and the Fund of the Chinese Academy of Tropical Agricultural Sciences (Project No. 1630062013018; 1630062015024).

'Corresponding author. E-mail: chenjiao52@163.com or yuandebao@163. com.
2004; Sakamoto et al., 2004). One interesting zinc finger transcription factor is the ring zinc finger domain protein, which was originally named after the acronym for the first RING finger gene (Joazeiro and Weissman, 2000) and was defined by the presence of a consensus sequence harboring cysteine (Cys) and histidine (His) residues (Cys-X2-Cys-X939-Cys-X1-3-His-X2-3-Cys/His-X2-Cys-X4-48-Cys-X2-Cys where $\mathrm{X}$ can be any amino acid) that can form a binding site for two zinc atoms. The canonical RING-finger protein family can be subcategorized into two types, RING-H2 and RING-HC, on the basis of the fifth coordination site, Cys or His, respectively. Additional modified types of RINGs, including RING-v, RING-D, RING-S/T, RING-G, and RING-C2, have been previously identified in the arabidopsis (Arabidopsis thaliana) genome (Kosarev et al., 2002; Stone et al., 2005). RING finger proteins play important roles in various biological processes (Ciechanover, 1998; Lyzenga and Stone, 2012), including photomorphogenesis (Ang and Deng, 1994), plant growth and development (Li et al., 2011; Zhang et al., 2008), and hormone signaling pathways (Bu et al., 2009; Zhang et al., 2007). In particular, RING finger proteins have been reported to be involved in negatively or positively regulating stress responses of multiple plants (Craig et al., 2009; Dong et al., 2006; Kim and Kim, 2013; Lee and Kim, 2011; Lee et al., 2009; Li et al., 2014; Liu et al., 2008; Ryu et al., 2010; Zhang et al., 2008). For 
Table 1. Primer sequences used for cloning and expression analysis of banana MaRING2 and stress-responsive genes in tobacco plants.

\begin{tabular}{lll}
\hline \multirow{2}{*}{ Names } & \multicolumn{1}{c}{ Forward } & Rences $\left(5^{\prime}-3^{\prime}\right)$ \\
\cline { 2 - 3 } MaRING2-5 ${ }^{\prime}$ RACE & & Reverse \\
MaRING2-Full & AACTGCTGCAAAGACACAGGT & GAGAAGGAGACCGAGAGAAGAC \\
MaRING2-qPCR & ATGGATGTCTTCTCTCGG & GATATCCCTTCTGCAAAGCG \\
MaActin1 & TGGAGAAACAGTGAGGAGATTACC & TCAGAAGAACCGTGGAGTATCG \\
NbWRKY17-qPCR & GAGGCACCAGTCCGTAGATAGC & CCGCCAGATGAAGGTACAACAT \\
NbMYB1-qPCR & CCGCTTTCTACTTCTTCA & GCTGGGACTCTAACAACTC \\
NbbZIP-qPCR & GTTACACGAAATGCTTGG & TTTGGAGTTCTGAGGAGG \\
NbUbiquitin & TAGGTCACGGATGAGGAA & ATTAGCATCAGCCCAAAA \\
\hline
\end{tabular}

${ }^{\mathrm{z}} \mathrm{RACE}=$ rapid amplification of cDNA ends; qPCR $=$ quantitative polymerase chain reaction.

example, a T-DNA insertion mutant of arabidopsis ATL9 resulted in increased susceptibility to powdery mildew (Erysiphe cichoracearum) (Ramonell et al., 2005). Constitutive expression of arabidopsis ATL2 exhibited the up-regulation and accumulation of defense-related salicylic acid- and jasmonic acid-responsive genes (Serrano and Guzman, 2004). Transgenic tobacco (Nicotiana tabacum) plants that constitutively express rice (Oryza sativa) RING gene OsBIRF1 exhibited enhanced disease resistance against Tobacco mosaic virus and oxidative stress tolerance via up-regulating the expression of oxidative stress-related genes (Liu et al., 2008). The chinese wild grapevine (Vitis pseudoreticulata) RING finger protein 1 (EIRP1) positively regulated plant disease resistance by mediating proteolysis of the negative regulator VpWRKY11 (Yu

et al., 2013). Constitutive expression of a hot pepper (Capsicum annuum) CaRmalH1 in tomato (Solanum lycopersicum) conferred strongly enhanced tolerance to drought and salt stresses (Seo et al., 2012). The RING finger gene NtRHF1 played a positive role in drought stress tolerance possibly through transcriptional regulation of several stress-responsive marker genes in tobacco (Xia et al., 2013). The RING finger protein STRF1 was involved in membrane trafficking and modulated salt-stress response in arabidopsis (Tian et al., 2015). The maize (Zea mays) RING-H2 finger protein gene ZmXERICO was upregulated in salt, drought, abscisic acid, and cold stresses (Gao et al., 2012). A Brassica rapa RING finger protein gene BrRZFP1 overexpression conferred increased tolerance to cold, salt, and dehydration stresses (Jung et al., 2013). Suppression of arabidopsis AtATL78 increased tolerance to cold stress (Kim and Kim, 2013), and MaRING1 functionally
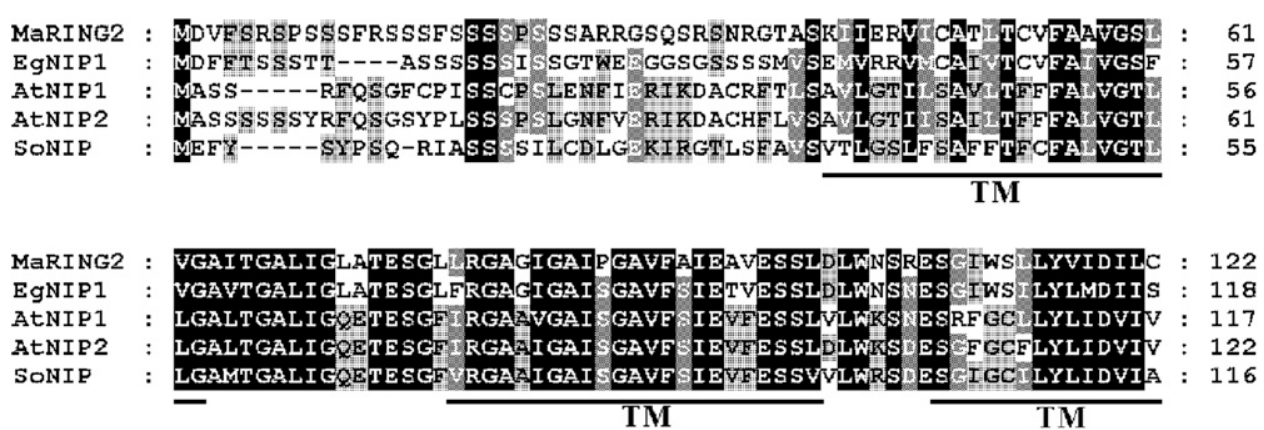

MARING2 : SLLSGRIVREKVDPAMQSAVQSQMSAADLLSIDNFDLFATDSTGGLAMDTVENLPKTNITA : 183 EgNIP1 : SLLSGRLVREKV CPAV SAVQSQMSAYD SP PME IP DFFET CGTGMSEDSVDKLPKIKI TA : 179 ATNIP1 : SLI SGRLVRERI CPAMLSAVQSQMGAVDT TEELSSTEDT GGSKGL GDLVDIIFKIKITG : 178 ALNIP2 : SLLSGRLVRERI CPAML SAVQSQMGAVDMAPDDHTSIFDT CGSKGL GDHVEKI PK T I TG : 183 SONIP : SLLSGRLVRERI PAMLSAVQSQMGA EDNGDITAIFDTESVRGL SGDVEKIPKIKITK : 177

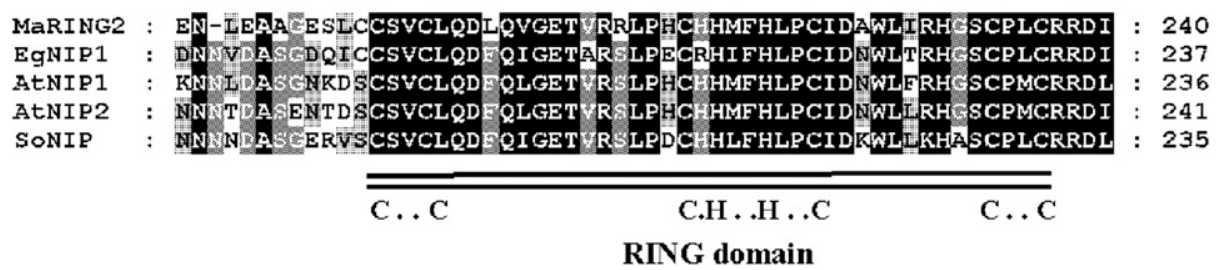

Fig. 1. Amino acid sequence alignment of the banana MaRING2 with other plant NIP RING-H2 finger proteins. MaRING2 was aligned with oil palm EgNIP1 (XP 010918134), arabidopsis AtNIP1 (At2g17750), arabidopsis AtNIP2 (Q8GT74), and spinach SoNIP (AM883105). Identical and similar amino acids were presented by black and gray shading, respectively. Gaps were introduced to optimize alignment. Simple bars below the sequences indicate the location of the three N-terminal transmembrane domains (TM). The C-terminal RING domain is double underlined, and the significant amino acids that determine the RING are noted below the line. coordinated with MaMYC2a in response to the cold stress of banana fruit (Chen et al., 2014).

Cold storage is an effective method that is used to prolong the postharvest qualities and extend the shelf life of a broad range of fruitbearing and horticultural products. However, tropical and subtropical fruits, such as banana, are highly susceptible to chilling injury (CI). Banana fruit stored at low temperatures $\left(<13{ }^{\circ} \mathrm{C}\right)$ generally results in peel pitting, discoloration, and abnormal fruit ripening, which significantly limit storage life and lead to substantial losses because of quality deterioration (Chen et al., 2008, 2011). Thus, it is important to understand the mechanism of $\mathrm{CI}$ in banana fruit and optimize methods that alleviate the symptoms. Our previous studies have shown that pretreatment with ABA before low temperature storage could enhance the chilling tolerance of banana fruit and thus alleviate CI (Wang et al., 2003, 2007). ABA is a well-known plant stress hormone (Tuteja, 2007; Xiong et al., 2002; Yamaguchi-Shinozaki 
and Shinozaki, 2006) and plays important roles in mediating adaptive responses to various environmental stresses, such as high salinity, drought, cold, and other abiotic stresses (Night and Knight, 2001). The pathways leading to stress adaptation can be divided into two major types: ABA-dependent and ABA-independent pathways (Himmelbach et al., 2003; Jakoby et al., 2002; Ladyzhenskaya, 2001; Zhu, 2002). During the ABA-mediated stress response, many stress-related genes, including various kinases and transcription factor genes, have been up-regulated under stress conditions (Chak et al., 2000; Choi et al., 2000; Kang et al., 2002; Koncz and Szabados, 1997; Seki, 2002; Zhu, 2002). Increasing evidence indicates that RING finger proteins play important roles in regulating ABA signaling and in related abiotic stress responses in plants (Lee et al., 2001; Lopez-Molina et al., 2003; Stone et al., 2006; Zhang et al., 2005, 2007). The arabidopsis RING-H2 finger protein gene $X E R I C O$ confers drought tolerance through enhanced ABA biosynthesis (Ko et al., 2006). Salt- and drought-induced ring fingerl (SDIRl) is an active E3 ligase and participates in ABA-mediated stress signal transduction (Zhang et al., 2007). Arabidopsis RING finger E3 ligase RHA2b acts additively with RHA2 $a$ in regulating ABA signaling and drought response (Li, 2011). These results suggest a link between RING finger proteins and ABA-mediated stress responses in plants. However, the functional relationship between ABA and RING finger proteins in response to chilling stress in banana fruit is far from being clearly understood.

In this study, a novel RING gene, MaRING2, was isolated and characterized from banana fruit. The expression patterns of MaRING2 related to ABA-induced chilling tolerance of banana fruit were investigated. Moreover, in contrast to the wild-type (WT) plants, MaRING2overexpressing transgenic tobacco plants (35S:MaRING2) showed hypersensitive phenotypes to exogenous ABA in terms of the germination rate, accumulated higher transcript levels of stress-responsive genes in response to cold tolerance, and were markedly tolerant to cold stress. Overall, the data presented in this study indicate that MaRING2, a C3H2C3-type RING protein, plays a role as a positive regulator in the ABA-dependent cold response in banana fruit.

\section{Materials and Methods}

PLANT MATERIALS AND TREATMENTS. Preclimacteric banana fruit at the $75 \%$ to $80 \%$ plump stage were obtained from a commercial plantation near Guangzhou, China. Banana fruit of uniform weight, shape, and maturity that were free of visual defects were used for this study. ABA (0.1 mM) treatment was performed as described previously (Chen et al., 2008), and the treated fruit were stored at $7^{\circ} \mathrm{C}$ (cold stress) and $90 \%$ relative humidity for $7 \mathrm{~d}$. For cold stress, fruit without ABA pretreatment were directly stored at $7{ }^{\circ} \mathrm{C}$, while for the non-chilling temperature control, fruit were directly stored at $22^{\circ} \mathrm{C}$. Samples were taken at 0,6 , and $12 \mathrm{~h}$ and $1,3,5$, and $7 \mathrm{~d}$, and banana peels were collected. The sampled peel tissues were frozen in liquid nitrogen immediately and stored at $-80{ }^{\circ} \mathrm{C}$ for further use. All treatments were repeated three times with three replicates.

The WT tobacco plants were used for phenotypic assays of 35S:MaRING2 plants in all experiments. Unless noted otherwise, plants were grown under sterile conditions on Murashige and Skoog (MS) nutrient agar medium containing $2 \%$ sucrose, or on soil in a growth chamber $(16 \mathrm{~h}$ light $/ 8 \mathrm{~h}$ dark $)$ at $23{ }^{\circ} \mathrm{C}$.

RNA EXTRACTION, GENE ISOLATION, AND SEQUENCE ANALYSIS. Frozen tissues were ground in liquid nitrogen using a mortar and pestle. Total RNA was extracted using the hot borate method (Wan and Wilkins, 1994). Potentially contaminating DNA was eliminated by treating the RNA with DNAse I using an RNAse-free kit (Promega, Madison, WI). The DNA-free total RNA was then used as a template for reverse transcription
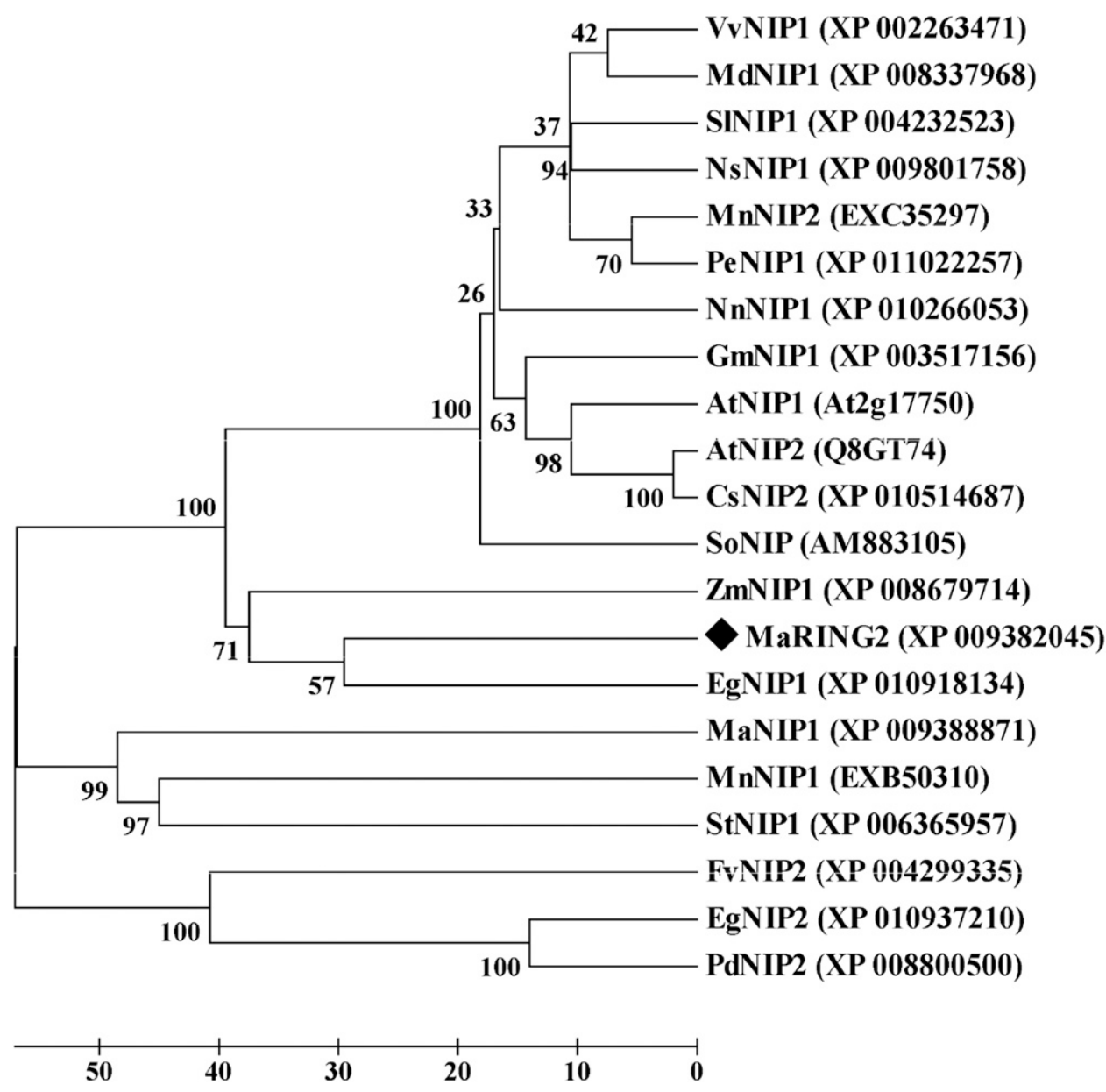

Fig. 2. Phylogenetic tree of banana MaRING2 with 20 other plant NIP RING-H2 finger proteins. Alignments were made using CLUSTAL X (version 1.83; Toby Gibson, Heidelberg, Germany) and the phylogenetic tree was constructed with MEGA 5.0 (version 5.0, Tokyo Metropolitan University, Tokyo, Japan) using a bootstrap test of phylogeny with minimum evolution test and default parameters. Numbers at the branch points indicated bootstrap values. A scale of distance is shown in the bottom. 
polymerase chain reaction (RT-PCR). The first-strand cDNA of the product was subjected to PCR amplification. The novel MaRING2 gene induced by cold stress was isolated from a transcriptome database obtained by RNA sequencing (RNA-seq). Briefly, harvested banana fruit at 0 and $1 \mathrm{~d}$ of cold storage at $7{ }^{\circ} \mathrm{C}$ were chosen and constructed into two different libraries. The library constructions and RNA-seq was performed using a high-throughput Solexa/Illumina sequencing platform, and sequences were assembled and annotated by the Beijing Genome Institute (BGI, Shenzhen, China), with the parameters described by Feng et al. (2012). One RING finger gene, induced by cold stress and belonging to the NIP family, was found in the transcriptome database. Thus, this RING finger gene, termed MaRING2, was selected, and its sequence was verified by recloning and resequencing. Then, the $5^{\prime}$-end sequence of the MaRING2 was obtained by $5^{\prime}$-rapid amplification of cDNA ends (RACE) using a RACE kit (TaKaRa Biotechnology, Dalian, China) according to the manufacturer's instructions, and the full sequence of MaRING2 was further confirmed by resequencing. The specific primers used for PCR amplification and RACE are shown in Table1.

A randomized complete block design was used with three replications. Identification of nucleotide sequences was performed using the National Center for Biotechnology Information (NCBI) Blast program (Altschul et al., 1997). Alignment and comparison of the sequences were performed with the Clustal W program (Thompson et al., 1994). Open reading frame (ORF) and protein predictions were performed using NCBI ORF Finder (Stothard, 2000). The theoretical isoelectric point $(\mathrm{pI})$ and mass values for mature peptides were calculated using the PeptideMass program (Gasteiger et al., 2003). A phylogenetic tree was constructed using the neighbor-joining method in the MEGA 5 (version 5.0; Tokyo Metropolitan University, Tokyo, Japan) program and was visualized by TreeView software (Page, 1996).

Expression analysis by Quantitative Real-time PCR. The transcript levels of MaRING2 in banana fruit under various treatments and expression patterns of stress-responsive genes in tobacco (WT and transgenic lines) before and after cold stress or ABA treatment were analyzed by quantitative real-time PCR (qRT-PCR) using the SYBR Green dye method. Synthesis of the first-strand cDNA and all qRT-PCR analyses were performed as described previously (Chen et al., 2011; Zhao et al., 2013). Actin or ubiquitin was used as an internal control to normalize the relative expression level of the analyzed genes in banana or tobacco, respectively. All qRT-PCR reactions were normalized using $C_{\mathrm{t}}$ values corresponding to the reference gene. The relative expression levels of the target gene were calculated with the formula $2^{-\Delta \Delta}$ CT (Livak and Schmittgen, 2001). Three independent biological replicates were used in the analysis. Primer sequences used for qRT-PCR analysis are listed in Table 1.

Plant transformation and generation of transgenic PLANTS. To construct a vector for plant transformation, the full-length cDNA of MaRING2 was amplified by PCR using an $X b a \mathrm{I}$ and KpnI primer set: PMV-RING1-UP GCtctagaATGGATGTCTTCTCTCGG and PMV-RING1-REV GGggtaccCTAGATATCCCTTCTGCAAAGCG. The PCR products were digested with $X b a \mathrm{I}$ and $K p n \mathrm{I}$ and inserted in the sense orientation into the $X b a \mathrm{I} / K p n \mathrm{I}$ sites of a plant expression vector, PMV $\left(\mathrm{Kan}^{\mathrm{r}}\right.$, CaMV 35Spromoter, NOS terminator, T DNA), to replace the $b$-glucuronidase (GUS) gene, under control of the CaMV $35 \mathrm{~S}$ promoter. After sequence confirmation the construct was introduced into agrobacterium strain EHA105. To produce transgenic tobacco plants, agrobacteriummediated transformation of leaf discs was carried out according to Horsh et al. (1985). The presence of the transgene in the kanamycin (Kan)-resistant seedlings was verified by PCR using primers: RG-UP: ATGGATGTCTTCTCTCGG; RGREV: GATATCCCTTCTGCAAAGCG. Overexpression of MaRING2 in two of the selected putative transgenic plants was examined by semiquantitative RT-PCR using the forward primer 5' -TGGAGAAACAGTGAGGAGATTACC-3' and the reverse primer 5' -TCAGAAGAACCGTGGAGTATCG-3', while the ubiquitin gene was used as an internal control (Table 1). $\mathrm{T} 2$ seeds of the overexpressing lines were harvested according to Liu et al. (2009) for the stress tolerance assay; the details are as follows: T0 transgenic plants were verified by PCR, and then T1 seeds were harvested, T1 transgenic plants, in which kanamycin-resistant segregation was 3:1, were considered for T2 seed harvesting.

ANALYSIS OF COLD TOLERANCE, ELECTROLYTE LEAKAGE AND MALONDIALDEHYDE. For cold treatment, each 10 seedlings of WT and transgenic plants were planted in the same pot filled with a mixture of soil and sand (1:1) where they were regularly watered for 1 month before the stress was induced. Then, the control (WT) and transgenic plants were chilled for $24 \mathrm{~h}$ at $2{ }^{\circ} \mathrm{C}$, after $24 \mathrm{~h}$ of treatment, the phenotypes were observed, the plants were photographed, the degree of the leaf blade wilting was observed, and the survival rate was calculated.

Malondialdehyde (MDA) concentration was measured as the indexes of membrane condition according to the previous studies with minor modifications (Chen et al., 2008). Leaf tissues $(2.0 \mathrm{~g})$ were homogenized in $6 \mathrm{~mL}$ of $10 \%$ trichloroacetic acid and then centrifuged for $15 \mathrm{~min}$ at $10,000 \mathrm{~g}_{\mathrm{n}}$. The supernatant was collected and $2 \mathrm{~mL}$ was mixed with $6 \mathrm{~mL}$ of $0.6 \%$ thiobarbituric acid. The mixture was heated to $100{ }^{\circ} \mathrm{C}$ for $20 \mathrm{~min}$, quickly cooled to $4^{\circ} \mathrm{C}$ and centrifuged at $10,000 g_{\mathrm{n}}$ for $10 \mathrm{~min}$. Absorbance of the supernatant was measured at 532,

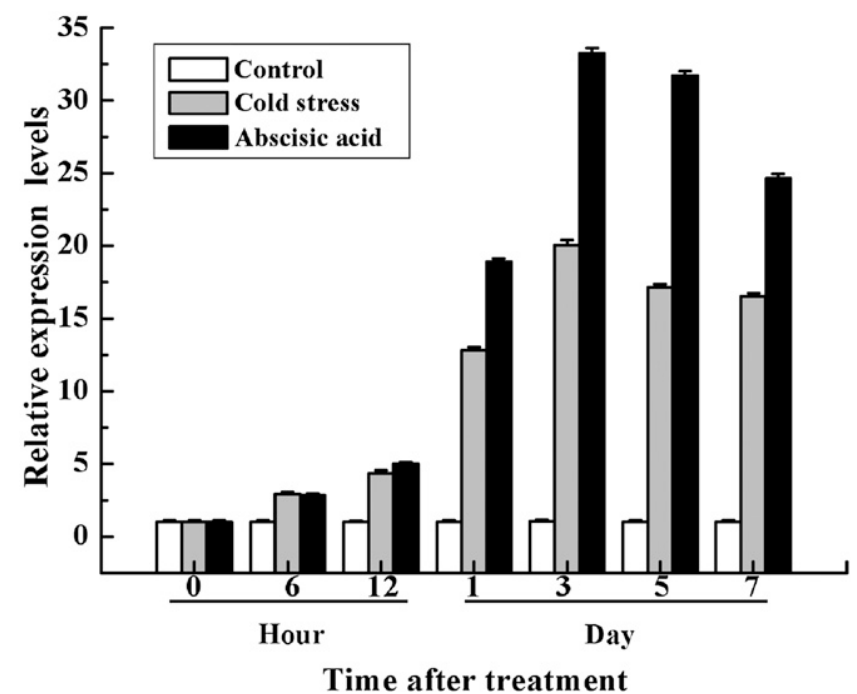

Fig. 3. Expression of MaRING2 in banana fruit during $7 \mathrm{~d}$ of storage. Abscisic acid (ABA)-treated banana fruit were stored at $7^{\circ} \mathrm{C}$ for $7 \mathrm{~d}$, for cold stress, fruit without ABA pretreatment were directly stored at $7{ }^{\circ} \mathrm{C}$, while for noncold stress control, fruit were directly stored at $22{ }^{\circ} \mathrm{C}$. Expression level at different time points was expressed as a ratio relative to the harvest time $(0 \mathrm{~d}$ of non-cold stress control), which was set at 1 . Each value represents the means of three biological replicates, and vertical bars indicate the SE. 
600, and $450 \mathrm{~nm}$ using an ultraviolet spectrophotometer (Shimadzu, Kyoto, Japan). MDA concentration was calculated according to the formula: $6.45 \times\left(A_{532}-A_{600}\right)-0.56 \times A_{450}$. Three replicates were used for each assessment.

The electrolyte leakage (EL) was measured as the indexes of membrane condition according to the method of Zhang and Ervin (2009) with some modifications. Fresh leaf discs (100 mg) were washed and then placed in Falcon tubes with $20 \mathrm{~mL}$ distilled water. The tubes were shaken for $24 \mathrm{~h}$ and an initial electrical conductance $(\mathrm{C} 1)$ was determined using a conductivity meter (model DDSJ_308A; Shanghai Precision and Scientific, Shanghai, China). The tubes were boiled for $20 \mathrm{~min}$, cooled down to $25^{\circ} \mathrm{C}$, and the final electrical conductance $(\mathrm{C} 2)$ was measured. The EL was calculated according to the formula: $\mathrm{EL}(\%)=(\mathrm{C} 1 / \mathrm{C} 2) \times 100$.

GERMINATION ASSAY. Germination assays were performed in triplicate with 25 to 30 seeds. The seeds of WT and $35 S$ : MaRING2 plants were cleansed with 3\% sodium hypochlorite for surface sterilization for $10 \mathrm{~min}$ in $2.0 \mathrm{~mL}$ centrifuge tube, and were cleansed with sterilized water six times, and then were imbibed in $0.05 \%$ agar solution in dark for $2 \mathrm{~d}$ at $4{ }^{\circ} \mathrm{C}$ for vernalization. Next, seeds were sown in MS medium containing $0,0.1,0.5$, or $1 \mu \mathrm{M}$ ABA (Sigma-Aldrich, St. Louis, MO) at $22{ }^{\circ} \mathrm{C}$ with a 16-h-light/ 8-h-dark photoperiod, the percentages of radicle emergence and cotyledon greening were determined after 3 and $7 \mathrm{~d}$, respectively.

\section{Results}

Cloning of a Full-Length CDNA OF THE MARING 2 GENE AND SEQUENCE ANALYSIS. In this study, a novel putative RING full-length cDNA was isolated from banana fruit and designated as MaRING2. MaRING2 was predicted to encode proteins of 240 amino acids, with a calculated molecular weight of $25.4 \mathrm{kDa}$ and a pI value of 5.72. A BLAST search of GenBank revealed that MaRING2 had $72 \%$ identity at the amino acid level with Elaeis guineensis NIP1. The multiple sequence alignment of NIPs showed that the deduced amino acid sequences of MaRING2 contained all of the structural features of the plant NIP RING-H2 finger proteins, three N-terminal transmembrane domains, and a C-terminal $\mathrm{C} 3 \mathrm{H} 2 \mathrm{C} 3$ type RING finger signature; the positions of these domains are underlined (Fig. 1). The phylogenetic tree analysis showed that MaRING2 clustered with EgNIP1 and ZmNIP1 and had the closest relationship with EgNIP1 (Fig. 2). These results suggest that MaRING2 encodes a RING-H2 finger protein with structural features common to the plant-specific NIP family.

MARING2 WAS UP-REgUlated BY COLD AND ABA. To understand the possible role of MaRING2 in ABA-induced banana fruit chilling tolerance, the expression pattern of MaRING2 in the peel of cold-stored fruit after ABA treatment was investigated by qRT-PCR. As shown in Fig. 3, compared with the expression of MaRING2 in non-cold control fruit, the expression of MaRING 2 in the fruit directly stored at $7{ }^{\circ} \mathrm{C}$ (cold stress) increased at $6 \mathrm{~h}$ and reached $\approx 18$-fold on day 3 , suggesting that MaRING2 was responsive to chilling and that the expression levels of MaRING2 were strongly induced by ABA treatment. The accumulation level in ABA-treated fruit was 13- and 14.5- fold higher than that in the cold stress fruit after 3 and $5 \mathrm{~d}$ of storage, respectively. These data indicate that MaRING2 is cold-inducible and might be related to ABA induced-chilling tolerance of banana fruit.

GENERATION AND SELECTION OF MARING2 TRANSGENIC TOBACCO PLANTS. MaRING2 was introduced into tobacco via agrobacterium-mediated transformation, and was constitutively
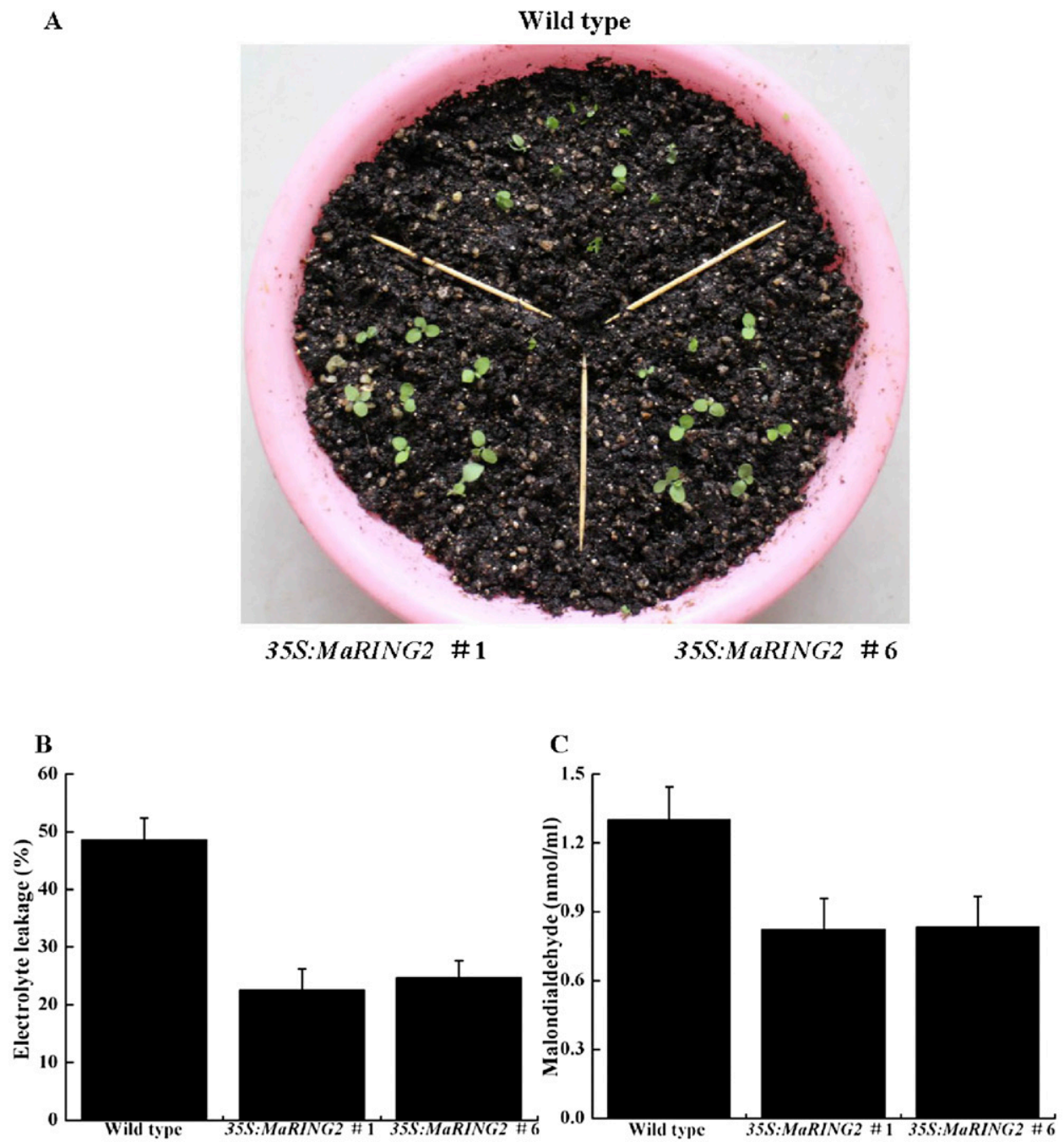

Fig. 4. Overexpression of banana MaRING2 enhances cold tolerance in transgenic tobacco. (A) The photographs are of representative tobacco wild-type (WT) and transgenic lines (35S:MaRING2 \#1 and 35S:MaRING2 \#6) after chilling for $24 \mathrm{~h}$. (B) Electrolyte leakage (EL) and (C) malondialdehyde (MDA) level in the WT, 35S: MaRING2 \#1, and 35S:MaRING2 \#6 after cold tolerance. Each value represents the means of three biological replicates, and vertical bars indicate the SE. 
expressed under the control of the CaMV35S promoter. To verify this integration, kan-resistant transgenic plants were analyzed using PCR. RT-PCR analysis showed that MaRING2 mRNA was detected in the transgenic plants, but not in the WT (data not shown). We selected nine transgenic lines harboring the MaRING2 transgene to monitor their expression in 3- to 4week-old plants (data not shown). Two of the lines (1 and 6), which had a higher MaRING2 expression level, were used for the stress tolerance test. None of these transgenic plants had any visible alterations from the WT morphology.

AsSESSMENT OF COLD TOLERANCE IN THE TRANSGENIC LINES. To assess the effect of MaRING2 overexpression on cold tolerance, 1-month-old WT and transgenic plants were exposed to $24 \mathrm{~h}$ of chilling at $2^{\circ} \mathrm{C}$. The degree of cold resistance differed significantly between the two genotypes.

As shown in Fig. 4A, the transgenic tobacco plant lines 1 and 6 showed enhanced tolerance and the WT plants were extremely sensitive to this stress, WT plants showed a higher degree of the leaf blade wilting compared with the transgenic plants, the WT plants survival rate was $40 \%$ (4 of 10), and the survival rate of the transgenic plant lines 1 and 6 were $80 \%$ (8 of 10 ) and $60 \%$ (6 of $10)$, respectively. In addition, EL, an important indicator of membrane injury, was significantly higher in the WT $(48.6 \%)$ than in line $1(22.5 \%)$ or $6(24.7 \%)$, indicating that the WT suffered from severe membrane damage (Fig. 4B). The MDA level exhibited a profile similar to the EL, which was significantly lower in the transgenic lines relative to the WT (Fig. 4C). These findings demonstrate that the transgenic lines were more resistant to cold stress.

EXPRESSION ANALYSIS OF STRESS-RESPONSIVE GENES IN THE WT AND TRANSGENIC LINES BEFORE AND AFTER COLD TOLERANCE. A number of plant transcription factors, such as $b Z I P, W R K Y$, and $M Y B$, have been shown to be involved in plant response to environmental stress (Lindemose et al., 2013). To gain further insight into the molecular mechanism underlying the enhanced cold resistance in the transgenic lines, the transcript abundance of stress-responsive genes (NbWRKY17, NbMYB1, and $N b b Z I P$ ) was examined in the WT and transgenic lines before and after $24 \mathrm{~h}$ of cold stress (Fig. 5). Under normal conditions (before cold stress), the mRNA levels of all three genes in lines 1 and 6 were higher than those in the WT, the accumulation level of $N b W R K Y 17$ in lines 1 and 6 was 0.9 - and 1.1-fold higher than that in the WT, the expression level of $N b M Y B 1$ in lines 1 and 6 was 1.6- and 2.1-fold higher than that in the WT, and the transcript level of NbbZIP in lines 1 and 6 was 1 - and 2.2-fold higher than that in the WT. Exposure to chilling caused up-regulation of the transcript levels of the analyzed genes in all lines, but lines 1 and 6 still had a higher expression level in comparison with the WT, the accumulation level of $N b W R K Y 17$ in lines 1 and 6 was 2.3- and 2.2-fold higher than that in the WT, the expression level of $N b M Y B 1$ in lines 1 and 6 was 1.0- and 2.0-fold higher than that in the WT, and the transcript level of NbbZIP in lines 1 and 6 was 3.2- and 4.4-fold higher than that in the WT. These results demonstrate that overexpression of MaRING2 in tobacco enhances the transcript levels of the stress-responsive genes with or without cold stress.

The 35S:MARING2 PLANT IS hypersensitive To ABA. We compared the seed germination rates of WT and 35S:MaRING2 plants in the presence or absence of ABA. To measure the germination rates, $\approx 25$ seeds were placed on full-strength solid MS medium supplemented with different concentrations $(0$, $0.1,0.5,1$, or $2 \mu \mathrm{M}$ ) of ABA. After $3 \mathrm{~d}$ of stratification, the
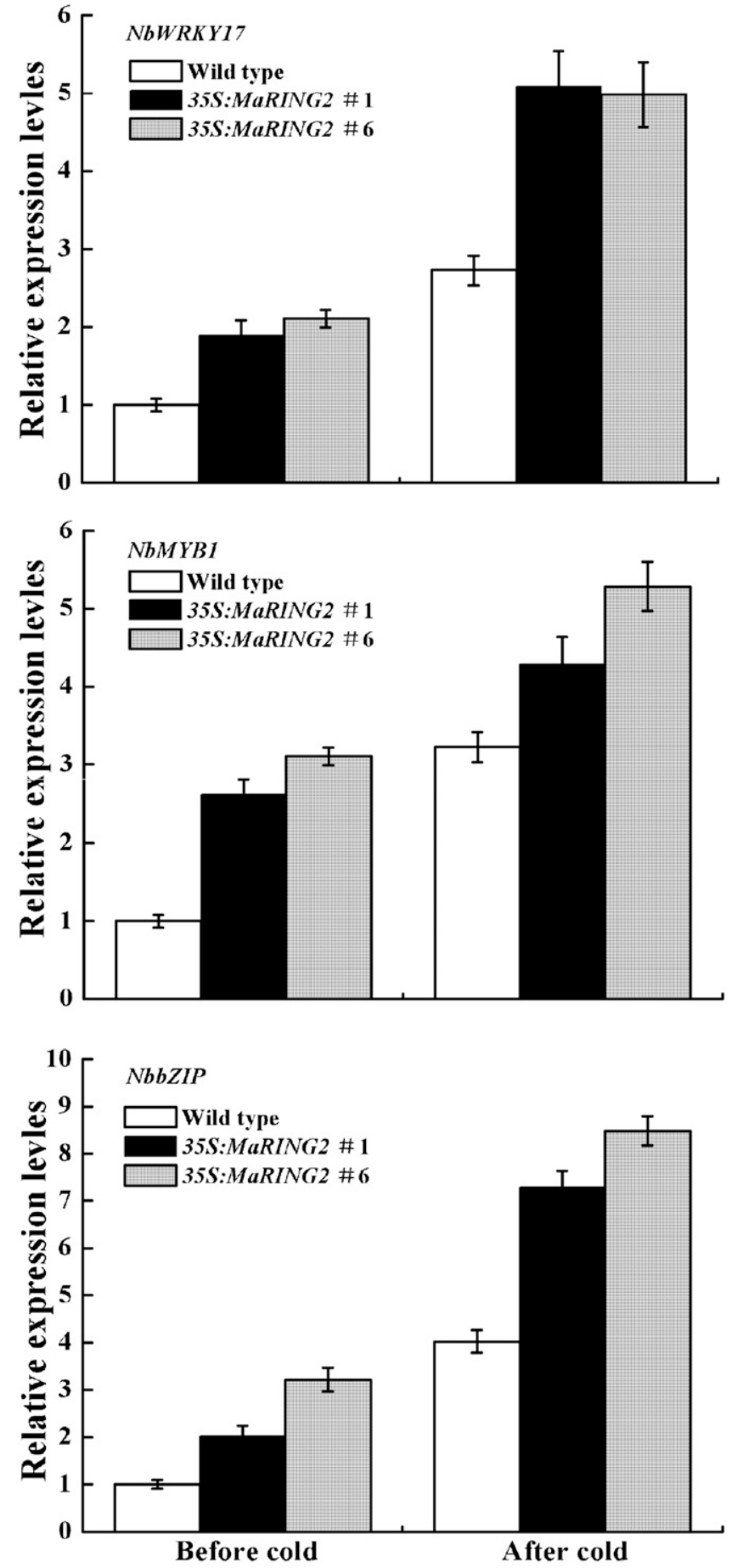

Fig. 5. Quantitative real-time polymerase chain reaction (PCR) analysis of expression levels of stress-responsive genes in the wild-type (WT) and the transgenic tobacco plants (35S:MaRING2 \#1 and 35S:MaRING2 \#6) under normal (before cold stress) and cold conditions (after $24 \mathrm{~h}$ of cold stress). Each value represents the means of three biological replicates, and vertical bars indicate the SE.

germination rates were determined as the percentage of radicle emergence (Fig. 6A). WT and 35S:MaRING2 (lines 1 and 6) seeds fully germinated on MS medium without ABA. On the ABA-containing growth medium, the germination rates of WT seeds were concomitantly reduced as the concentration of ABA increased. In the presence of $0.5 \mu \mathrm{M} \mathrm{ABA}, 56 \%$ of WT seeds failed 
A

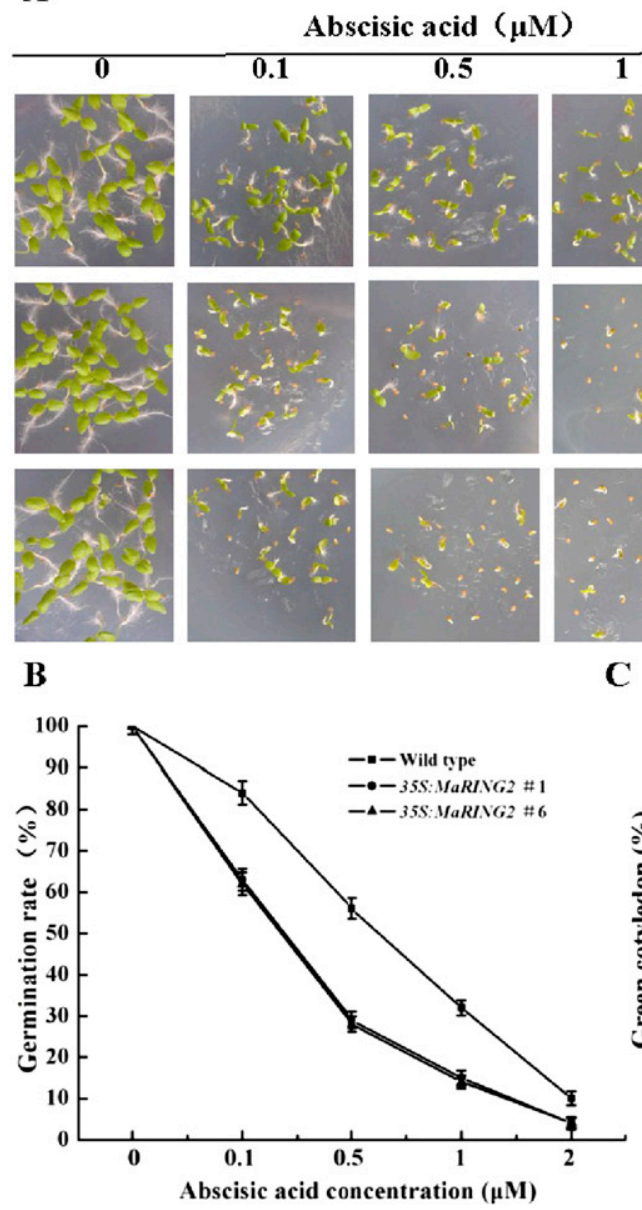

C

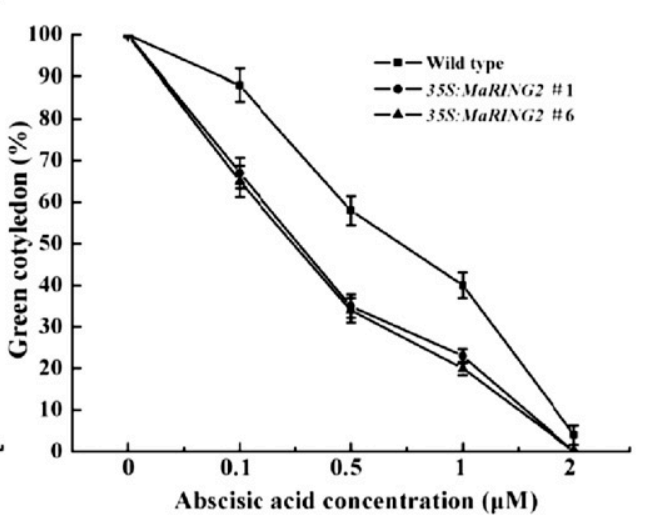

Fig. 6. Phenotypes of tobacco wild-type (WT) and transgenic lines (35S:MaRING2 \#1 and 35S:MaRING2 \#6) in response to abscisic acid (ABA) during seed germination and early seedling growth. (A) ABA sensitivity of WT, 35S:MaRING2 \#1, and 35S:MaRING2 \#6 plants in the germination stage. Surface-sterilized seeds were imbibed in $0.05 \%$ agar solution for $2 \mathrm{~d}$ at $4{ }^{\circ} \mathrm{C}$ for vernalization, sown on Murashige and Skoog (MS) medium supplied with $\mathrm{ABA}(0,0.1,0.5,1$, and $2 \mu \mathrm{M})$, and then incubated for $7 \mathrm{~d}$ at $22{ }^{\circ} \mathrm{C}$ under a 16 -h-light/8-h-dark photoperiod; bars $=0.5 \mathrm{~cm}$. (B) Quantification of radicle emergence in response to ABA. (C) Quantification of cotyledon greening in response to ABA. Average and SD values were determined from three biological replicates $(n=25-30)$.

Stone et al., 2006; Zhang et al., 2005, 2007), auxin (Xie et al., 2002), and brassinosteroid (Molnar et al., 2002). In particular, ABA is an essential mediator in triggering plant responses to common abiotic stresses, including drought, salt, heat, cold, and oxidative stresses (Finkelstein et al., 2002; Xie et al., 2002). However, up to now, the report of the function of NIPs was that AthATL25/NIP2 mediated the intraplastidial trafficking of RPOTmp at thylakoid membranes (Guzmán, 2012). Whether NIP RING-H2 proteins have additional functions related to ABA-mediated abiotic stress responses has never been explored. Therefore, in this study, we attempted to investigate the functional relationship between ABA and NIP RING-H2finger protein in response to cold stress in banana fruit.

We report here the role of the MaRING2 gene that encoded a NIP RING-H2 finger protein (Figs. 1 and 2) and played a positive role in regulating ABA signaling and stress responses. MaRING2 was coldinducible, and its transcript levels increased rapidly following the ABA treatment during cold storage (Fig. 3). In addition, overexpression of MaRING2 led to ABA-associated phenotypes such as ABA hypersensitivity in terms of radicle emergence and cotyledon development (Fig. 6), reduced EL and the MDA level (Fig. 4B and C), enhanced the

to germinate at $3 \mathrm{~d}$ after culture (Fig. 6B). In contrast, 35S: MaRING2 transgenic seeds exhibited hypersensitivity to ABA. More than $50 \%$ of these transgenic seeds were unable to germinate in the presence of $0.5 \mu \mathrm{M}$ ABA, and less than $5 \%$ of the transgenic seeds germinated with $2 \mu \mathrm{M}$ ABA (Fig. 6B). In addition, at $7 \mathrm{~d}$ after germination, $\approx 40 \%$ of WT seedlings developed true green cotyledons in the presence of $1 \mu \mathrm{M}$ ABA (Fig. 6C). In contrast, under the same concentration of $\mathrm{ABA}$, the growth of $77 \%$ and $80 \%$ of $35 S$ :MaRING2 (lines 1 and 6 ) transgenic seedlings was completely arrested and true green cotyledons failed to develop at $7 \mathrm{~d}$ after germination (Fig. 6C). Thus, MaRING2-overexpressing plants were hypersensitive to $\mathrm{ABA}$ in terms of both radicle emergence and cotyledon development.

\section{Discussion}

A wide variety of $R I N G$ finger genes were isolated from multiple plant species, such as arabidopsis, rice, and poplar (Populus tremula), which contain 469, 378, and 399 RING finger genes, respectively (Du et al., 2009; Kraft et al., 2005). Some RING finger proteins are involved in the regulation of hormone signaling pathways, including ABA (Bu et al., 2009; transcript levels of the ABA-mediated abiotic stress response genes (Galbiati et al., 2011; Jiang et al., 2014; Suzuki et al., 2003; Xiang et al., 2008; Yan et al., 2014; Ying et al., 2012), NbWRKY17, NbMYB1, and NbbZIP (Fig. 5), and, therefore, increased cold tolerance (Fig. 4A). These are supported by previous studies which reported that the maize RING-H2 finger protein gene $Z m X E R I C O$ was up-regulated in salt, drought, abscisic acid, and cold stress (Gao et al., 2012); overexpression of RING finger protein gene BrRZFPl conferred increased tolerance to cold, salt, and dehydration stresses (Jung et al., 2013); and overexpression of SDIR 1 and $R H A 2 b$ led to ABA hypersensitivity and ABA-associated phenotypes, such as salt hypersensitivity in germination, enhanced ABA-induced stomatal closing, and enhanced drought tolerance (Li, 2011; Zhang et al., 2007). These data support that MaRING2 is a positive regulator of an ABA-dependent response to cold stress.

It is noteworthy that our analyses suggest that MaRING2 may mediate the degradation of substrates that are negative regulators of ABA signaling. Alternatively, MaRING2 may mediate the stabilization and activation of substrates that are positive regulators of ABA signaling. Future identification of MaRING2 substrates will further our understanding of the ABA signaling pathway. 


\section{Literature Cited}

Altschul, S.F., T. Madden, A. Schaffer, J. Zhang, Z. Zhang, W. Miller, and D.J. Lipman. 1997. Gapped BLAST and PSI-BLAST: A new generation of protein database search programs. Nucl. Acids Res. 25:3389-3402.

Ang, H.L. and X.W. Deng. 1994. Regulatory hierarchy of photomorphogenic loci: Allele-specific and light-dependent interaction between the HY5 and COP1 loci. Plant Cell 6:613-628.

$\mathrm{Bu}, \mathrm{Q}$., H. Li, and Q. Zhao. 2009. The arabidopsis RING finger E3 ligase RHA2a is a novel positive regulator of abscisic acid signaling during seed germination and early seedling development. Plant Physiol. 150:463-481.

Chak, R.K., T.L. Thomas, R.S. Quatrano, and C.D. Rock. 2000. The genes ABI1 and ABI2 are involved in abscisic acid- and droughtinducible expression of the Daucus carota L. Dc3 promoter in guard cells of transgenic Arabidopsis thaliana (L.) Heynh. Planta 210:875-883.

Chen, J., J.F. Kuang, W. Shana, J.N. Wang, Y.Y. Xiao, J.Y. Chen, and W.J. Lu. 2014. Molecular characterization of a cold-responsive RING-H2 finger gene from banana fruit and its interaction with MaMYC2a. Postharvest Biol. Technol. 98:48-55.

Chen, J.Y., L.H. He, Y.M. Jiang, Y. Wang, D.C. Joyce, Z.L. Ji, and W.J. Lu. 2008. Role of phenylalanine ammonia-lyase in heat pretreatment-induced chilling tolerance in banana fruit. Physiol. Plant. 132:318-328.

Chen, L., H.Y. Zhong, J.F. Kuang, J.G. Li, W.J. Lu, and J.Y. Chen. 2011. Validation of reference genes for RT-qPCR studies of gene expression in banana fruit under different experimental conditions. Planta 234:377-390.

Choi, H., J. Hong, J. Ha, J. Kang, and S.Y. Kim. 2000. ABFs, a family of ABA-responsive element binding factors. J. Biol. Chem. 275:1723-1730.

Ciechanover, A. 1998. The ubiquitin-proteasome pathway: On protein death and cell life. EMBO J. 17:7151-7160.

Craig, A., R. Ewan, J. Mesmar, V. Gudipati, and A. Sadanandom. 2009. E3 ubiquitin ligases and plant innate immunity. J. Expt. Bot. 60:1123-1132.

Cui, J., G. Jander, L.R. Racki, P.D. Kim, N.E. Pierce, and F.M. Ausubel. 2002. Signals involved in arabidopsis resistance to Trichoplusia ni caterpillars induced by virulent and avirulent strains of the phytopathogen Pseudomonas syringae. Plant Physiol. 129:551-564.

Dong, C.H., M. Agarwal, Y.Y. Zhang, Q. Xie, and J.K. Zhu. 2006. The negative regulator of plant cold responses, HOS1, is a RING E3 ligase that mediates the ubiquitination and degradation of ICE1. Proc. Natl. Acad. Sci. USA 21:8281-8286.

Du, Z., X. Zhou, L. Li, and Z. Su. 2009. Plants UPS: A database of plants' ubiquitin proteasome system. BMC Genomics 10:227-233.

Feng, C., M. Chen, C.J. Xu, L. Bai, X.R. Yin, X. Li, A.C. Allan, I.B. Ferguson, and K.S. Chen. 2012. Transcriptomic analysis of chinese bayberry (Myrica rubra) fruit development and ripening using RNASeq. BMC Genomics 13:19-34.

Finkelstein, R.R., S.S. Gampala, and C.D. Rock. 2002. Abscisic acid signaling in seeds and seedlings. Plant Cell 14:S15-S45.

Galbiati, M., J.T. Matus, P. Francia, F. Rusconi, P. Cañón, C. Medina, L. Conti, E. Cominelli, C. Tonelli, and P. Arce-Johnson. 2011. The grapevine guard cell-related VvMYB60 transcription factor is involved in the regulation of stomatal activity and is differentially expressed in response to ABA and osmotic stress. BMC Plant Biol. 11:142-156.

Gao, Y., H. Li, D.X. Deng, S.Q. Chen, W. Jiang, and J.M. Chen. 2012. Characterization and expression analysis of the maize RING-H2 finger protein gene ZmXERICO responsive to plant hormones and abiotic stresses. Mol. Biol. Rep. 39:6513-6519.

Gasteiger, E., A. Gattiker, C. Hoogland, I. Ivanyi, R.D. Appel, and A. Bairoch. 2003. ExPASy: The proteomics server for in-depth protein knowledge and analysis. Nucl. Acids Res. 31:3784-3788.

Guzmán, P. 2012. The prolific ATL family of RING-H2 ubiquitin ligases. Plant Signal. Behav. 7:1014-1021.
Himmelbach, A., Y. Yang, and E. Grill. 2003. Relay and control of abscisic acid signaling. Curr. Opin. Plant Biol. 6:470-479.

Horsh, R.B., J.E. Fry, N.L. Hoffmann, D. Eichholtz, S.C. Rogers, and R.T. Fraley. 1985. A simple and general method for transferring genes into plants. Science 227:1229-1231.

Huang, J., J.F. Wang, Q.H. Wang, and H.S. Zhang. 2005. Identification of a rice zinc finger protein whose expression is transiently induced by drought, cold but not by salinity and abscisic acid. Mitochondrial DNA 16:130-136.

Jakoby, M., B. Weisshaar, W.D. Laser, J.V. Carbajosa, J. Tiedemann, T. Kroj, and F. Parcy. 2002. bZIP transcription factors in arabidopsis. Trends Plant Sci. 7:106-111.

Jiang, Y.Z., Y.J. Duan, J. Yin, S.L. Ye, J.R. Zhu, F.Q. Zhang, W.X. Lu, D. Fan, and K.M. Luo. 2014. Genome-wide identification and characterization of the Populus WRKY transcription factor family and analysis of their expression in response to biotic and abiotic stresses. J. Expt. Bot. 65:6629-6644.

Joazeiro, C.A. and A.M. Weissman. 2000. RING finger proteins: Mediators of ubiquitinligase activity. Cell 102:549-552.

Jung, Y.J., I.H. Lee, I.S. Nou, K.D. Lee, A.M. Rashotte, and K.K. Kang. 2013. BrRZFP1 a Brassica rapa C3HC4-type RING zinc finger protein involved in cold, salt and dehydration stress. Plant Biol. 15:274-283.

Kang, J.Y., H.I. Choi, M.Y. Im, and S.Y. Kim. 2002. Arabidopsis basic leucine zipper proteins that mediate stress-responsive abscisic acid signaling. Plant Cell 14:343-357.

Kim, J.C., S.H. Lee, Y.H. Cheng, C.M. Yoo, S.I. Lee, H.J. Chun, D.J. Yun, J.C. Hong, S.Y. Lee, C.O. Lim, and M.J. Cho. 2001. A novel cold inducible zinc-finger protein from soybean, SCOF-1, enhances cold tolerance in transgenic plants. Plant J. 25:247-259.

Kim, S.J. and W.T. Kim. 2013. Suppression of arabidopsis RING E3 ubiquitin ligase AtATL78 increases tolerance to cold stress and decreases tolerance to drought stress. FEBS Lett. 587:2584-2590.

Ko, J.H., S.H. Yang, and K.H. Han. 2006. Upregulation of an arabidopsis RING-H2 gene, XERICO, confers drought tolerance through increased abscisic acid biosynthesis. Plant J. 47:343355.

Koncz, C. and L. Szabados. 1997. Differential expression of two P5CS genes controlling proline accumulation during salt-stress requires $\mathrm{ABA}$ and is regulated by $\mathrm{ABA} 1, \mathrm{ABI} 1$ and $\mathrm{AXR} 2$ in arabidopsis. Plant J. 12:557-569.

Kosarev, P., K.F. Mayer, and C.S. Hardtke. 2002. Evaluation and classification of RING-finger domains encoded by the Arabidopsis genome. Genome Biol. 3:0016.1-0016.12.

Kraft, E., S.L. Stone, L. Ma, N. Su, Y. Gao, O. Lau, X. Deng, and J. Callis. 2005. Genome analysis and functional characterization of the E2 and ring-type E3 ligase ubiquitination enzymes of arabidopsis. Plant Physiol. 139:1597-1611.

Ladyzhenskaya, E.P. 2001. Pathways of the abscisic acid hormonal signal transduction across the plant cell plasma membrane. Membr. Cell Biol. 14:699-713.

Lee, H., L. Xiong, Z. Gong, M. Ishitani, B. Stevenson, and J.K. Zhu. 2001. The arabidopsis HOS1 gene negatively regulates cold signal transduction and encodes a RING finger protein that displays coldregulated nucleo-cytoplasmic partitioning. Genes Dev. 15:912-924.

Lee, H.K., S.K. Cho, and O. Son. 2009. Drought stress-induced Rma1H1, a RING membrane-anchor E3 ubiquitin ligase homolog, regulates aquaporin levels via ubiquitination in transgenic arabidopsis plants. Plant Cell 21:622-641.

Lee, J.H. and W.T. Kim. 2011. Regulation of abiotic stress signal transduction by E3ubiquitin ligases in arabidopsis. Mol. Cells 31:201-208.

Li, H. 2011. The arabidopsis RING finger E3 ligase RHA2b acts additively with RHA2a in regulating abscisic acid signaling and drought response. Plant Physiol. 156:550-563.

Li, J.T., N. Wang, L.N. Wang, H.P. Xin, and S.H. Li. 2014. Molecular cloning and characterization of the HOS1 gene from 'Muscat Hamburg' grapevine. J. Amer. Soc. Hort. Sci. 139:54-62. 
Li, Y., B. Wu, Y. Yu, G. Yang, C. Wu, and C. Zheng. 2011. Genomewide analysis of the RING finger gene family in apple. Mol. Genet. Genomics 286:81-94.

Lindemose, S., C. O'Shea, M.K. Jensen, and K. Skriver. 2013. Structure, function and net-works of transcription factors involved in abiotic stress responses. Intl. J. Mol. Sci. 14:58425878.

Liu, H.Z., H.J. Zhang, Y.Y. Yang, G.J. Li, Y.X. Yang, X. Wang, B.M. Basnayake, D.Y. Li, and F.M. Song. 2008. Functional analysis reveals pleiotropic effects of rice RING-H2 finger protein gene OsBIRF1 on regulation of growth and defense responses against abiotic and biotic stresses. Plant Mol. Biol. 68:17-30.

Liu, X., Z. Wang, L. Wang, R. Wu, J. Phillips, and X. Deng. 2009. LEA 4 group genes from the resurrection plant Boea hygrometrica confer dehydration tolerance in transgenic tobacco. Plant Sci. 176:90-98.

Livak, K.J. and T.D. Schmittgen. 2001. Analysis of relative gene expression data using real-time quantitative PCR and the 2(-Delta Delta C (T)) method. Methods 25:402-408.

Lopez-Molina, L., S. Mongrand, N. Kinoshita, and N.H. Chua. 2003. AFP is a novel negative regulator of ABA signaling that promotes ABI5 protein degradation. Genes Dev. 17:410-418.

Lyzenga, W.J. and S.L. Stone. 2012. Abiotic stress tolerance mediated by protein ubiquitination. J. Expt. Bot. 63:599-616.

Molnar, G., S. Bancos, and F. Nagy. 2002. Characterisation of brassinosteroid-responsive RING-H2 gene from Arabidopsis thaliana. Planta 215:127-133.

Mukhopadhyay, A., S. Vii, and A.K. Tyagi. 2004. Overexpression of a zinc finger protein gene from rice confers tolerance to cold, dehydration, and salt stress in transgenic tobacco. Proc. Natl. Acad. Sci. USA 101:6309-6314.

Night, H. and M.R. Knight. 2001. Abiotic stress signalling pathways: Specificity and crosstalk. Trends Plant Sci. 6:262-267.

Page, R.D. 1996. TreeView: An application to display phylogenetic trees on personal computers. Comput. Appl. Biosci. 124:357-358.

Ramonell, K., M. Berrocal-Lobo, S. Koh, J. Wan, H. Edwards, G. Stacey, and S. Somerville. 2005. Loss-of-function mutations in chitin responsive genes show increased susceptibility to the powdery mildew pathogen Erysiphe cichoracearum. Plant Physiol. 138:1027-1036.

Ronald, P. and H. Leung. 2002. The rice genome: The most precious things are not jade and pearls. Science 296:58-59.

Ryu, M.Y., S.K. Cho, and W.T. Kim. 2010. The arabidopsis C3H2C3type RING E3 ubiquitinligase AtAIRP1 is a positive regulator of an abscisic acid-dependent response to drought stress. Plant Physiol. 154:1983-1997.

Sakamoto, H., K. Maruyama, Y. Sakuma, T. Meshi, M. Iwabuchi, K. Shinozaki, and K. Yamaguchi-Shinozaki. 2004. Arabidopsis Cys2/ His2-type zinc-finger proteins function as transcription repressors under drought, cold and high-salinity stress conditions. Plant Physiol. 1136:2734-2746.

Seki, M. 2002. Monitoring the expression profiles of 7000 Arabidopsis genes under drought, cold and high-salinity stresses using a fulllength cDNA microarray. Plant J. 31:279-292.

Seo, Y.S., J.Y. Choi, S.J. Kim, E.Y. Kim, J.S. Shin, and W.T. Kim. 2012. Constitutive expression of CaRma1H1, a hot pepper ERlocalized RING E3 ubiquitin ligase, increases tolerance to drought and salt stresses in transgenic tomato plants. Plant Cell Rpt. 31:16591665.

Serrano, M. and P. Guzman. 2004. Isolation and gene expression analysis of Arabidopsis thaliana mutants with constitutive expression of ATL2, an early elicitor-response RING-H2 zinc-finger gene. Genetics 167:919-929.

Stone, L.S., H. Hauksdottir, A. Troy, J. Herschleb, E. Kraft, and J. Callis. 2005. Functional analysis of the RING-type ubiquitin ligase family of Arabidopsis. Plant Physiol. 137:13-30.

Stone, S.L., L.A. Williams, L.M. Farmer, R.D. Vierstra, and J. Callis. 2006. Keep on going, a RING E3 ligase essential for arabidopsis growth and development, is involved in abscisic acid signaling. Plant Cell 18:3415-3428.

Stothard, P. 2000. Sequence Manipulation Suite. 2 Feb. 2010. <http:// www.bioinformatics.org/sms/Index.html>.

Suzuki, M., M.G. Ketterling, Q.B. Li, and D.R. McCarty. 2003. Viviparous1 alters global gene expression patterns through regulation of abscisic acid signaling. Plant Physiol. 132:1664-1677.

Thomashow, M.F. 1999. Plant cold acclimation: Freezing tolerance genes and regulatory mechanism. Annu. Rev. Plant Physiol. Plant Mol. Biol. 50:571-599.

Thompson, J.D., D.G. Higgins, and T.J. Gibson. 1994. CLUSTAL W: Improving the sensitivity of progressive multiple sequence alignment through sequence weighting, position-specific gap penalties and weight matrix choice. Nucl. Acids Res. 22:4673-4680.

Tian, M., L.J. Lou, L.J. Liu, F.F. Yu, Q.Z. Zhao, H.W. Zhang, Y.R. Wu, S.Y. Tang, R. Xia, B.G. Zhu, G. Serino, and Q. Xie. 2015. The RING finger E3 ligase STRF1 is involved in membrane trafficking and modulates salt-stress response in Arabidopsis thaliana. Plant J. 82:81-92.

Tuteja, N. 2007. Abscisic acid and abiotic stress signaling. Plant Signal. Behav. 2:135-138.

Wan, C.Y. and T.A. Wilkins. 1994. A modified hot borate method significantly enhances the yield of high quality RNA from cotton (Gossypium hirsutum L.). Anal. Biochem. 223:7-12.

Wang, Y., J.Y. Chen, Y.M. Jiang, and W.J. Lu. 2007. Cloning and expression analysis of phenylalanine ammonia-lyase in relation to chilling tolerance in harvested banana fruit. Postharvest Biol. Technol. 44:34-41.

Wang, Y., W.J. Lu, Z.Q. Zhang, and H. Xie. 2003. ABA and putrescine treatments alleviate chilling injury in banana fruits during storage at $8^{\circ}$ C. J. Plant Physiol. Mol. Biol. 29:549-554.

Xia, Z.L., X.H. Su, J.J. Liu, and M.P. Wang. 2013. The RING-H2 finger gene 1 (RHF1) encodes an E3 ubiquitin ligase and participates in drought stress response in Nicotiana tabacum. Genetics 141:11-21.

Xiang, Y., N. Tang, H. Du, H. Ye, and L. Xiong. 2008. Characterization of OsbZIP23 as a key player of the basic leucine zipper transcription factor family for conferring abscisic acid sensitivity and salinity and drought tolerance in rice. Plant Physiol. 148:19381952.

Xie, Q., H.S. Guo, and G. Dallman. 2002. SINAT5 promotes ubiquitinrelated degradation of NAC1 to attenuate auxin signals. Nature 419:167-170.

Xiong, L., K.S. Schumaker, and J.K. Zhu. 2002. Cell signaling during cold, drought, and salt stress. Plant Cell 14:S165-S183.

Xiong, L. and J.K. Zhu. 2002. Molecular and genetic aspect of plant responses to osmotic stresses. Plant Cell Environ. 25:131-139.

Yamaguchi-Shinozaki, K. and K. Shinozaki. 2006. Transcriptional regulatory networks in cellular responses and tolerance to dehydration and cold stresses. Annu. Rev. Plant Biol. 57:781-803.

Yan, H., H. Jia, X. Chen, L. Hao, H. An, and X. Guo. 2014. The cotton WRKY transcription factor GhWRKY17 functions in drought and salt stress in transgenic Nicotiana benthamiana through ABA signaling and the modulation of reactive oxygen species production. Plant Cell Physiol. 55:2060-2076.

Ying, S., D.F. Zhang, J. Fu, Y.S. Shi, Y.C. Song, T.Y. Wang, and Y. Li. 2012. Cloning and characterization of a maize bZIP transcription factor, ZmbZIP72, confers drought and salt tolerance in transgenic arabidopsis. Planta 235:253-266.

Yu, Y., W.R. Xu, J. Wang, L. Wang, W.K. Yao, Y.Z. Yang, Y. Xu, F. Ma, Y.J. Du, and Y.J. Wang. 2013. The chinese wild grapevine (Vitis pseudoreticulata) E3 ubiquitin ligase Erysiphe necator-induced RING finger protein 1 (EIRP1) activates plant defense responses by inducing proteolysis of the VpWRKY11 transcription factor. New Phytol. 200:834-846.

Zhang, X., V. Garreton, and N.H. Chua. 2005. The AIP2 E3 ligase acts as a novel negative regulator of ABA signaling by promoting $\mathrm{ABI} 3$ degradation. Genes Dev. 19:1532-1543.

Zhang, Y., S. Feng, F. Chen, H. Chen, J. Wang, C. McCall, Y. Xiong, and X. Deng. 2008. Arabidopsis DDB1-CUL4 associated factor1 
forms a nuclear E3 ubiquitin ligase with DDB1 and CUL4 that is involved in multiple plant developmental processes. Plant Cell 20:1437-1455.

Zhang, Y.Y., C.W. Yang, Y. Li, N.Y. Zheng, H. Chen, Q.Z. Zhao, T. Gao, H. Guo, and Q. Xie. 2007. SDIR1 is a RING finger E3 ligase that positively regulates stress-responsive abscisic acid signaling in Arabidopsis. Plant Cell 19:1912-1929.

Zhao, M.L., J.N. Wang, W. Shan, J.G. Fan, J.F. Kuang, K.Q. Wu, X.P. Li, W.X. Chen, F.Y. He, J.Y. Chen, and W.J. Lu. 2013. Induction of jasmonate signalling regulators MaMYC2s and their physical interactions with MaICE1 in methyl jasmonateinduced chilling tolerance in banana fruit. Plant Cell Environ. $36: 30-51$

Zhu, J.K. 2001. Cell signaling under salt, water and cold stresses. Curr. Opin. Biotechnol. 9:214-219.

Zhu, J.K. 2002. Salt and drought stress signal transduction in plants. Annu. Rev. Plant Biol. 53:247-273.

Zhang, X. and E.H. Ervin. 2009. Physiological assessment of cool season turf grasses under ultraviolet-B stress. HortScience 44:17851789. 